\title{
Bichat's buccal fat pad removal: cheek reduction surgery
}

\begin{abstract}
In the past few years there have been a growing number of procedures for the removal of Bichat's buccal fat pad, also called cheek reduction surgery. Bichat's buccal fat pad may be used as part of the therapeutic procedure in cases of oroantral communication, peri-orbital defects, congenital cleft palate, and plastic surgery of facial recontouring. The purpose of this case report was to demonstrate the procedure of Bichat's buccal fat pad, due to the frequent habit of biting the oral mucosa by the patient. Buccal fat pad removal is a minor procedure, and the surgical technique is considered simple and safe if performed by trained and experienced professionals. The postoperative can be compared to an extraction of third molar, and the use of analgesics, anti-inflammatory controls properly any painful symptoms. The buccal fat pad removal should be carried out following a precise indication, always with the patient conscious of the risks and benefits that may be obtained.
\end{abstract}

Keywords: bichat's fat pad, buccal fat pad, cheek reduction
Volume 7 Issue 4 - 2017

\author{
Caio Vinicius G Roman Torres, ${ }^{1,2}$ Adhmar \\ Sani Junior, ${ }^{3}$ Juliana Cordeiro, ${ }^{4}$ Sergio \\ Marigny Filho, ${ }^{3}$ Rui Manuel Freire Sampaio, ${ }^{2,3}$ \\ Leticia C Cidreira Boaro,' Angelica Castro \\ Pimentel' \\ 'Professor of Department of Post Graduation, Division of \\ Implantology, School of Dentistry, University of Santo Amaro- \\ UNISA, Brazil \\ 2Professor of Department of Graduation, Division of Dentistry, \\ University Metropolitan Santos-UNIMES, Brazil \\ ${ }^{3}$ School of Dentistry, Department of Oral and Maxillofacial \\ Surgery and Traumatology, University of Sao Paulo (USP), Brazi \\ ${ }^{4}$ School of Dentistry, University of Santo Amaro-UNISA, Brazil
}

Correspondence: Caio Vinicius G Roman Torres, Professor of Departament of Post Graduation, Division of Implantology, School of Dentistry, University of Santo Amaro- UNISA, Sao Paulo, Brazil, Email cvgrt@hotmail.com

Received: May 16, 2017 | Published: June 21, 2017

\section{Introduction}

The adipose tissue of the cheeks has a lobed mass formed by a central body and four extensions: buccal, pterygoid, pterigo maxillary and temporal. The body is centrally positioned and is located above the parotid duct, behind the zygomatic arch and is divided into three lobes: anterior, intermediate and posterior in accordance with the structure of the bones, ligaments and blood vessels. The anterior lobe is located below the zygomatic, and extends to the front of the buccinator muscle, jaw and deep space square upper lip muscle. The parotid gland passes through the back, and the anterior facial vein passes through the anterior inferior margin. The anterior lobe also involves the orbital vessels. The ramification of the facial nerve are located on the outer surface of the fat pad capsule. The function of Bichat's buccal fat pad is to protect the sensitive anatomical structures around as vessels and nerve trauma and participates in functions of chewing and sucking especially in children. ${ }^{1-3}$ In the past few years, it has increased the number of procedures for removing the Bichat's buccal fat pad with aesthetic purpose, and both the plastic surgeon and the dental surgeon can perform it. The cheek reduction surgery or simply the surgery to narrow down the cheek is a surgical procedure that removes all or part of buccal fat pad..$^{4-8}$ A patient candidate for this type of surgery typically has an excessive facial circumference; in some cases lacerate the tissue of the buccal mucosa due to constant bites at the this location. The purpose of this case report was to demonstrate the buccal fat pad removal procedure in a patient who had frequent lesions on the jugal mucosa due to frequent bites and caused a better contour of the face after undergoing bariatric surgery and reduced weight in about $70 \mathrm{~kg}$.

\section{Case presentation}

Female patient, 28 years old, was referred to the dental care in surgery specialty, indicating the technique of cheek reduction surgery. Patient was not aesthetically satisfied because of a rounded face and constant bites at the oral mucosa. After completing the anamnesis and oral clinical assessment, the patient underwent laboratory tests for preoperative evaluation (complete blood count, fasting blood glucose and coagulation). After planning, we opted for treatment with removal of buccal fat pad bilaterally. To access buccal fat pad a small incision was done (Figure 1), no more than $2 \mathrm{~cm}$ long, into the soft tissue located in the lower portion of the zygomatic process taking care to properly see the orifice of the parotid gland. After this incision, dissection of the fat pad was achieved with a thin or hemostatic scissors, which is located under the zygomatic arch (Figure 2). It is very important to preserve the fascial lining of the fat pad, it will allow the removal of the hole fat pat at once. With a long hemostat tweezer inserted into the fat area, a portion of the fat pad is pinched drawn out. Gradually, the entire fat pad is pulled out with the help of another hemostat tweezer until the pedicle can be visualized. At this point, the pedicle can be cut and the fat pad can be loosed (Figure 3). A small suction tip can be inserted into the area to clean any part of the fat that may have been left during divulsion. A simple suture is carried closing the incision in the majority of cases. The postoperative care includes intensive cryotherapy within 48 hours, analgesics and anti-inflammatories. In the case presented, the patient showed a higher edema on the left (Figure 3). When the fat pad is not removed in a single piece the antibiotic therapy must be held for 5 to 7 days. The results can be observed 4 to 6 months after surgery. 


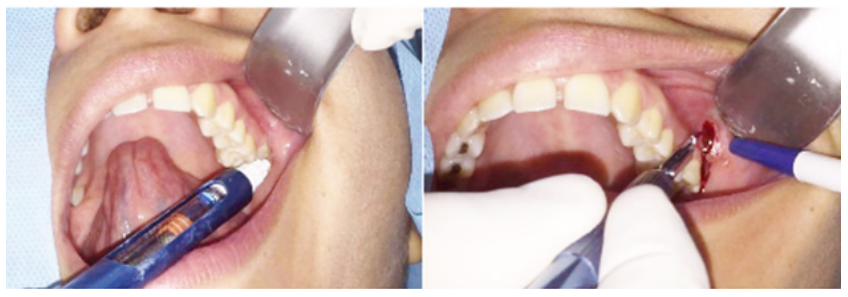

Figure I Infiltrative anesthesia/Incision in the oral mucosa.

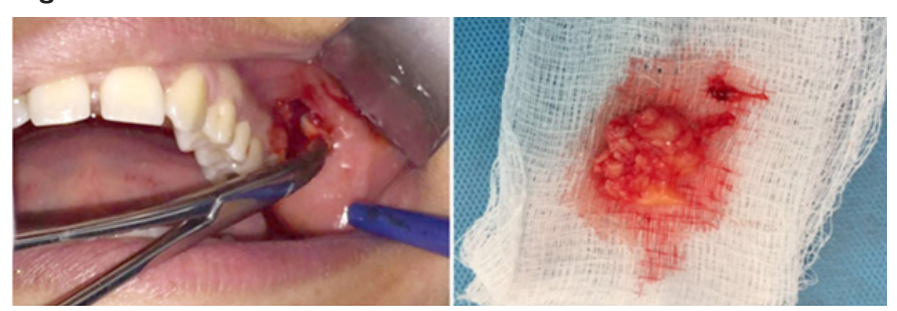

Figure 2 Dissection/Bichat's fat removal.

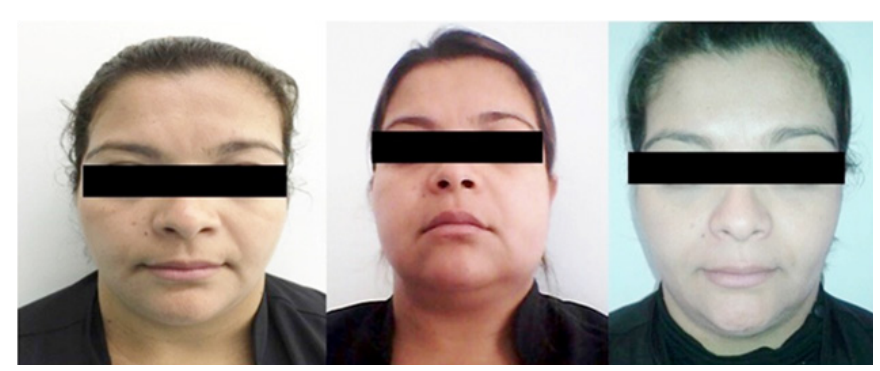

Figure 3 Initial/Post operative 3 days/Post operative 30 days. Discussion

The purpose of cheek reduction surgery is: reduce the volume of the face of the bottom of promoting thinning chin. However, this surgery is controversial for some professionals, because this face region usually lose fat with increasing age and the removal of those fat pockets can result in a more aged appearance, especially if the removal is total. It is important to note that throught the years, the face begins to suffer a loss of fat. Therefore, people who undergo this surgery may need fills later to regain a more youthful appearance. It is a purely aesthetic surgery and can be carried out by people who want to fine-tune the face. However, only present good results for people who normally stay with the larger circumferentially face when they are overweight. ${ }^{7}$ It is important a clinical evaluation by the professional who will perform the surgery so that he can diagnose whether there is indication and what are the expectations of the patient and the treatment. Among the postoperative complications more often related are hematomas, partial necrosis, infection or injury to the facial nerve ${ }^{8}$ exaggerated incisions can leave the tissue without adequate blood supply leading to necrosis. The preservation of the fascia overlying the fat pad promotes improved prognosis and reduces surgical time, the disruption would need suction and curettage of the area. The region where the buccal fat pad are located is close to two of the trigeminal nerve ramifications, the maxillary ramification (which follows the entire upper jaw) and mandibular ramification (which follows the lower jaw). Like many other nerves present in the face, the trigeminal nerve is a sensory nerve that controls sensation spreading across the face, sending them messages to the brain. ${ }^{9}$ If the professional is inexperienced and damage this nerve it can cause facial paralysis. In the case shown in this study, intra oral healing was observed in 15 days, and the end of facial edema after 4 weeks, which was the same reported in the literature. ${ }^{10}$ The procedure can be performed by a dentist as long as the practitioner has a good local anatomical knowledge, follows the indication for each case, and take all pre and postoperative care that is necessary to avoid complications.

\section{Funding}

None.

\section{Acknowledgments}

None.

\section{Conflicts of interest}

The author declares that there is no conflict of interest.

\section{References}

1. Brucoli M, Arcuri F, Borello G, et al. Surgical technique of the transoral approach to remove a lipoma of the buccal fat pad. J Craniofac Surg. 2011;22(6):2415-2418.

2. Zhang YP, Qi KM, Wang JQ, et al. Anatomical structure of the buccal fat pad and its clinical adaptations. Plastic Reconstructive Surg. 2002;109(7):2509-2518.

3. Egyedi P. Utilization of buccal fat pad for closure of oroantral and/or oronasal communications. J Maxillofacial Surg. 1977;5(4):241-244.

4. Shrivastava G, Padhiary S, Pathak H, et al. Buccal Fat Pad to Correct Intraoral Defects. Int J Sci Res Public. 2013;3(2):1-4.

5. Holton LH, Rodriguez ED, Silverman RP, et al. The buccal fat pad flap for periorbital reconstruction: a cadaver dissection and report of two cases. Plastic Reconstructive Surg. 2004;114(6):1529-1533.

6. Alkan A, Dolanmaz D, Uzun E,et al. The reconstruction of oral defects with buccal fat pad. Swiss Med Wkly. 2003;133(33-34):465-470.

7. Matarasso A. Buccal fat pad excision: aesthetic improvement of the midface. Ann Plast Surg. 1991;26(5):413-418.

8. Rohrich RJ, Pessa JE. The fat compartments of the face: anatomy and clinical implications for cosmetic surgery. Plast Reconstr Surg. 2007;119(7):2219-2227.

9. Hwang K, Cho HJ, Battuvshin D, et al. Interrelated buccal fat pad with facial buccal branches and parotid duct. J Craniofacial Surg. 2005;16(4):658-660.

10. Ramirez OM. Buccal fat pad pedicle flap for midface augmentation. Ann Plast Surg. 1999;43(2):109-118. 